\title{
Permutações Simétricas em Messiaen: uma Abordagem Interdisciplinar entre Matemática e Música
}

\author{
Symmetric Permutation in Messiaen: an Interdisciplinary \\ Approach between Mathematics and Music
}

\author{
Luciana Gastaldi S. Souza \\ Universidade Estadual de Londrina \\ lucianagastaldi@uel.br
}

\section{Tadeu Moraes Taffarello}

Universidade Estadual de Campinas tadeumt@unicampl.br

\section{Naresh Kumar Sharma \\ Universidade Estadual de Londrina \\ nsharma@uel.br}

Resumo: $\mathrm{O}$ trabalho analisa a técnica que o compositor Olivier Messiaen utilizou para trabalhar o ritmo em algumas de suas composições, a qual ele denominou de "permutações simétricas". Seriam estas permutações simétricas sob a ótica da geometria? Com o intuito de responder esta pergunta, discorreu-se sobre permutação e posteriormente sobre simetria. Percebeu-se que as permutações utilizadas por Messiaen não têm, necessariamente, as propriedades que as designariam simétricas, em termos geométricos. O que ele fazia, na realidade, era trabalhar as permutações dentro de um grupo, de maneira a conseguir variedade rítmica, porém dentro de certa limitação. Revela-se, dessa forma, como a matemática pode ser uma ferramenta poderosa no auxílio aos compositores e a importância de se ter uma visão interdisciplinar, sem fronteiras sólidas entre as áreas do conhecimento, rompendo as suas próprias lógicas, enriquecendo e indo além de conhecimentos específicos.

Palavras-chave: Matemática; Música; Interdisciplinaridade; Permutação; Simetria; Messiaen. 
Abstract: Oliver Messiaen developed a specific technique to operate with rhythmic parameters in some of his compositions. This paper focus on the analysis of this technique, which he called "symmetric permutations." Were these permutations considered symmetric when explored under the lens of geometry? In order to answer this question, this paper deals with permutation and later, with symmetry. In geometric terms, it was noticed that permutations used by Messiaen do not necessarily show the properties that designate symmetry. What he did, in fact, was to explore permutations within a group in order to achieve rhythmic variety, although with certain limitations. Consequently, it was revealed that mathematics can be a powerful tool in assisting composers. In addition, this paper shows how an interdisciplinary vision can break solid boundaries between different areas of expertise in order to create a new knowledge, enriching and going beyond its specificity.

Keywords: Mathematics; Music; Interdisciplinary; Permutation; Symmetry; Messiaen.

\section{1 - Introdução}

Olivier Messiaen foi um dos mais importantes compositores da segunda metade do século $X X$. Isso se deve, sobretudo, a três fatores principais:

1. A sua obra composicional compreende diversos gêneros musicais para as mais variadas instrumentações, desde peças para instrumentos solo, em especial o órgão ou o piano, até peças para grande orquestra, coro e solistas.

2. Em sua carreira, trabalhou cerca de 35 anos como professor de estética, harmonia e composição no Conservatório de Música de Paris, onde teve como alunos Pierre Boulez, Karlheinz Stockhausen, Iannis Xenakis, Gérard Grisey, Tristan Murail e Almeida Prado, dentre outros.

3. Publicou livros sobre música nos quais, além de analisar diversos autores da bibliografia musical existente, com especial destaque para Stravinsky, Mozart e Ravel, descreve várias das técnicas composicionais empregadas em suas próprias peças. Dentre estes livros, destacam-se as suas Vingt Leçons d'Harmonie, o seu Technique de mon langage musical e, principalmente, o seu Traité de rythme, de couleur et d'ornithologie com sete tomos em oito volumes.

E é justamente em seu Traité que Messiaen cunha o termo "Permutações Simétricas" relacionando-o a uma técnica específica de trabalhar o ritmo em suas composições. Percebe-se que Messiaen, ao utilizar o termo, relacionou áreas distintas da matemática. 
A permutação é um dos tópicos estudados em Análise Combinatória. Porém, o autor não a usa apenas como parte desta área da Matemática, ele aborda o conjunto das permutações como tendo a estrutura de um Grupo. Dessa maneira, para entendermos o que o autor entende por Permutação, é necessária a utilização da Teoria de Grupos.

O conceito de Simetria é utilizado na matemática em várias áreas. Justificaremos o termo utilizado por Messiaen como sendo este um tópico da Geometria.

Assim sendo, procederemos nosso estudo discorrendo inicialmente sobre Permutação e posteriormente sobre Simetria para estudarmos a possibilidade de unir as duas ideias, conforme proposto por Messiaen.

\section{2 - Permutação}

Seja $J_{n}$ o conjunto $J_{n}=\{1,2,3, \ldots, n\}$, com $n \in \mathbb{N}$.

Tomemos, como exemplo, $\mathrm{n}=3$, de modo que $\mathrm{J}_{3}=\{1,2,3\}$, o qual denotaremos simplesmente por J. Uma permutação de J é uma aplicação bijetiva f de J sobre J. Seria natural indicá-la por meio da tabela:

\begin{tabular}{c|c|c|c}
$N$ & 1 & 2 & 3 \\
\hline$f(n)$ & $f(1)$ & $f(2)$ & $f(3)$
\end{tabular}

Entretanto, é mais comumente indicada pela seguinte notação:

$$
f=\left(\begin{array}{ccc}
1 & 2 & 3 \\
f(1) & f(2) & f(3)
\end{array}\right)
$$

Seja $\mathrm{P}(\mathrm{J})$ o conjunto das permutações dos elementos de J. $\mathrm{P}(\mathrm{J})$ é fechado em relação à operação composição, pois:

$$
f, g \in \mathrm{P}(\mathrm{J}) \Rightarrow \text { sua composta } f \text { o } g \text { também está em } \mathrm{P}(\mathrm{J})
$$

Ou seja:

$$
\begin{gathered}
\mathrm{P}(\mathrm{J}) \mathrm{X} \mathrm{P}(\mathrm{J}) \rightarrow \mathrm{P}(\mathrm{J}) \\
(f, g) \rightarrow f \circ g
\end{gathered}
$$

$P(J)$, munido desta operação composição, é um grupo, pois satisfaz as seguintes propriedades: 
- Associatividade:

$$
f o(g o h)=(f \circ g) o h, \quad \forall f, g, h \in \mathrm{P}(\mathrm{J})
$$

- Elemento neutro, a saber, $I$, em que $I$ é a aplicação identidade. Se

$$
I: J \rightarrow J, \operatorname{com} \mathrm{I}(\mathrm{n})=\mathrm{n}, \quad \forall n \in J,
$$

então

$$
f o I=I o f=f, \quad \forall f \in \mathrm{P}(\mathrm{J})
$$

- Elemento Inverso: Se $f$ pertence ao conjunto das permutações $\mathrm{P}(\mathrm{J})$, claramente

$$
\begin{array}{r}
f^{1}: J \rightarrow J \\
n \rightarrow m
\end{array}
$$

também pertence.

Neste caso, $m$ é o único tal que $n=f(m)$. A existência deste $m$ é garantida, pois $f$ é sobrejetora. A unicidade deste $m$ é garantida, pois $f$ é injetora.

Seja $S_{1}$ o grupo das permutações do conjunto $\{1,2,3\}$.

Como o conjunto $\{1,2,3\}$ possui 3 elementos, o número de permutações é $3 !=6$.

São elas:

$$
\begin{aligned}
& f_{0}=\left(\begin{array}{lll}
1 & 2 & 3 \\
1 & 2 & 3
\end{array}\right), f_{1}=\left(\begin{array}{lll}
1 & 2 & 3 \\
2 & 3 & 1
\end{array}\right), f_{2}=\left(\begin{array}{lll}
1 & 2 & 3 \\
3 & 1 & 2
\end{array}\right) \\
& f_{3}=\left(\begin{array}{lll}
1 & 2 & 3 \\
1 & 3 & 2
\end{array}\right), f_{4}=\left(\begin{array}{lll}
1 & 2 & 3 \\
3 & 2 & 1
\end{array}\right), f_{5}=\left(\begin{array}{lll}
1 & 2 & 3 \\
2 & 1 & 3
\end{array}\right) .
\end{aligned}
$$

A composição de permutações pode ser apresentada segundo uma tabela (tábua do grupo), a qual é apresentada na Tabela 1, a seguir.

\begin{tabular}{|c|c|c|c|c|c|c|}
\hline \hline${ }^{\circ}$ & $\boldsymbol{f o}_{0}$ & $\boldsymbol{f}_{\mathbf{1}}$ & $\boldsymbol{f}_{2}$ & $\boldsymbol{f}_{3}$ & $\boldsymbol{f}_{4}$ & $\boldsymbol{f}_{5}$ \\
\hline \hline $\boldsymbol{f}_{0}$ & $f_{0}$ & $f_{1}$ & $f_{2}$ & $f_{3}$ & $f_{4}$ & $f_{5}$ \\
\hline $\boldsymbol{f}_{1}$ & $f_{1}$ & $f_{2}$ & $f_{0}$ & $f_{5}$ & $f_{3}$ & $f_{4}$ \\
\hline $\boldsymbol{f}_{2}$ & $f_{2}$ & $f_{0}$ & $f_{1}$ & $f_{4}$ & $f_{5}$ & $f_{3}$ \\
\hline $\boldsymbol{f}_{3}$ & $f_{3}$ & $f_{4}$ & $f_{5}$ & $f_{0}$ & $f_{1}$ & $f_{2}$ \\
\hline $\boldsymbol{f}_{4}$ & $f_{4}$ & $f_{5}$ & $f_{3}$ & $f_{2}$ & $f_{0}$ & $f_{1}$ \\
\hline $\boldsymbol{f}_{5}$ & $f_{5}$ & $f_{3}$ & $f_{4}$ & $f_{1}$ & $f_{2}$ & $f_{0}$ \\
\hline
\end{tabular}

Tabela 1: Tábua de $S_{1}$

\section{MUSICA THEORICA}


A leitura é feita linha por coluna. Por exemplo,

$$
f_{2}^{\circ} f_{1}=\left(\begin{array}{lll}
1 & 2 & 3 \\
3 & 1 & 2
\end{array}\right) \circ\left(\begin{array}{lll}
1 & 2 & 3 \\
2 & 3 & 1
\end{array}\right)=\left(\begin{array}{lll}
1 & 2 & 3 \\
1 & 2 & 3
\end{array}\right)=f_{0}
$$

Observe que ao elemento 1 corresponde o 3 e ao 3 o 1 novamente (segunda matriz). Portanto, ao 1 a composição faz corresponder o 1, e assim por diante.

$$
f_{2} \circ f_{2}=\left(\begin{array}{lll}
1 & 2 & 3 \\
3 & 1 & 2
\end{array}\right) \circ\left(\begin{array}{lll}
1 & 2 & 3 \\
3 & 1 & 2
\end{array}\right)=\left(\begin{array}{lll}
1 & 2 & 3 \\
2 & 3 & 1
\end{array}\right)=f_{1}
$$

Da mesma maneira foram obtidos os outros resultados.

\section{1. - Ordem de um elemento de um grupo}

Seja $a$ um elemento de um grupo G. Dizemos que $a$ tem ordem finita se existir um número natural não nulo $m$, tal que $a^{m}=1$. O menor natural não nulo com tal propriedade é chamado ordem de a e é indicado por $o(a)$.

\section{2. - Estudo de Messiaen com 12 elementos rítmicos}

Olivier Messiaen (1996, p. 12-13) usa números de 1 a 12 para representar um cromatismo rítmico, relacionando cada figura musical ao total de semicolcheias contidas nesta figura. Desse modo, 1 representa a semicolcheia, 2 a colcheia, 3 a colcheia pontuada e assim por diante, conforme a Figura 1 a seguir.

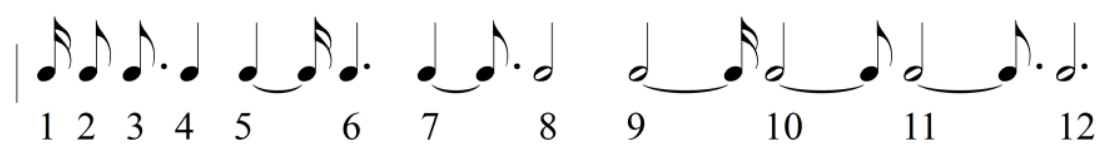

Figura 1: Representação numérica de um cromatismo rítmico com 12 elementos.

(cf. Messiaen 1996, p. 12)

A permutação que leva esta configuração nela mesma é a identidade $\left(f_{0}\right)$.

$$
f_{0}=\left(\begin{array}{llllllllllll}
1 & 2 & 3 & 4 & 5 & 6 & 7 & 8 & 9 & 10 & 11 & 12 \\
1 & 2 & 3 & 4 & 5 & 6 & 7 & 8 & 9 & 10 & 11 & 12
\end{array}\right)=I
$$

Com esse material, Messiaen escolhe uma permutação de modo que o primeiro elemento seja uma semicolcheia e os posteriores sigam uma progressão aritmética de razão 3, de 4 em 4 elementos.

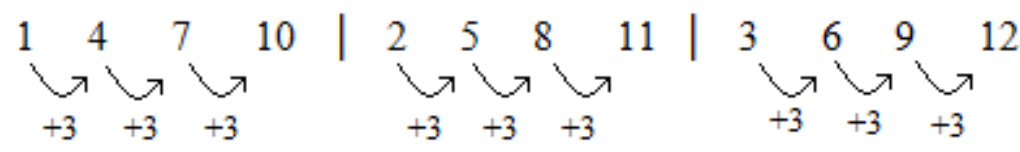


Permutação escolhida:

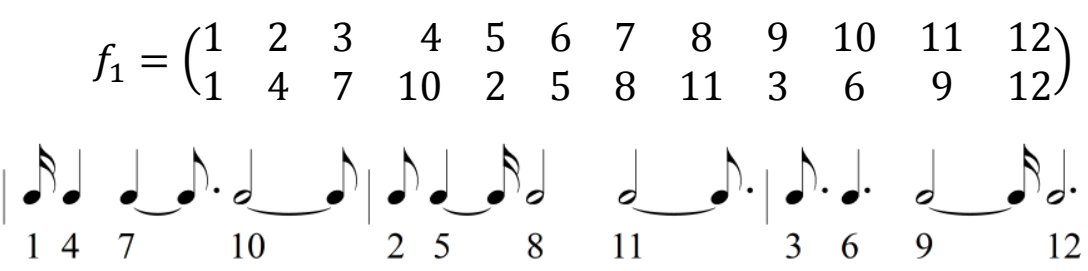

Figura 2: Permutação escolhida, em figuras musicais (cf. Messiaen 1996, p.12)

Iterando essa permutação, teremos:

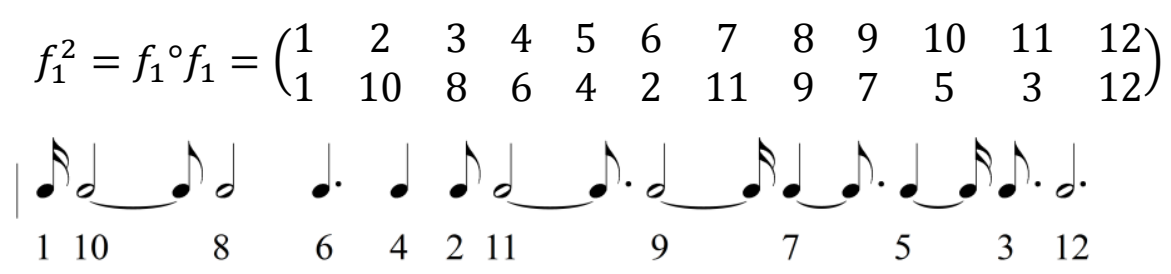

Figura 3: Iteração 2 da permutação escolhida (cf. Messiaen 1996, p.12)

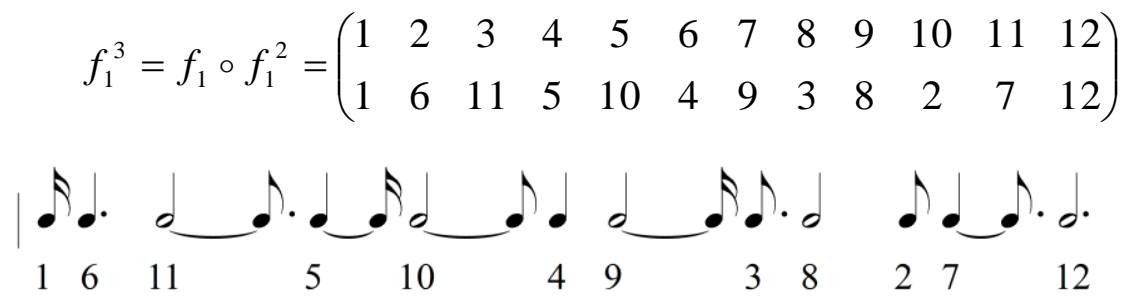

Figura 4: Iteração 3 da permutação escolhida (cf. Messiaen 1996, p.13)

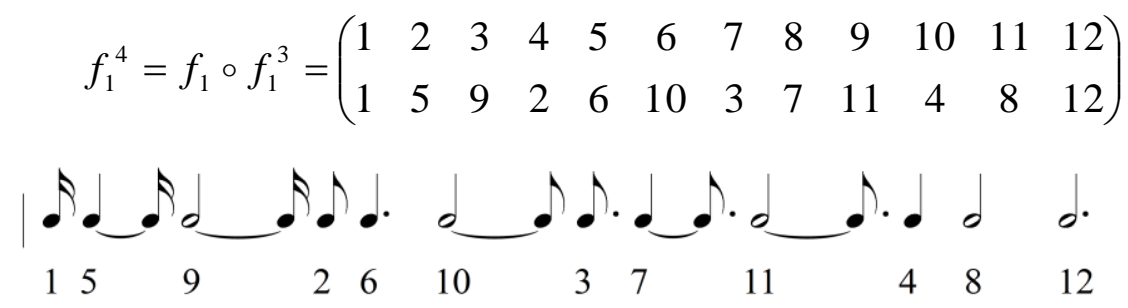

Figura 5: Iteração 4 da permutação escolhida (cf. Messiaen 1996, p.13)

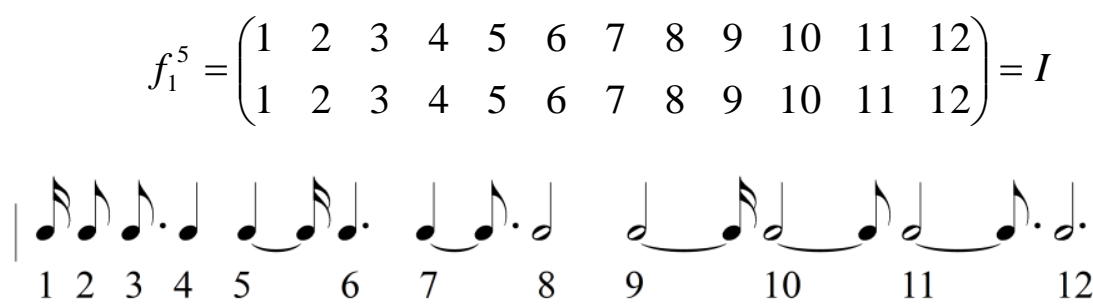

Figura 6: Iteração 5 da permutação escolhida (cf. Messiaen 1996, p.13) 
Percebe-se, desse modo, que a permutação escolhida é de ordem 5. Essa permutação foi usada como um estudo para uma composição maior na qual Messiaen utilizou um cromatismo rítmico de 32 figuras, da fusa à semibreve, a qual será detalhada no próximo tópico.

Definiremos, a seguir, ciclo.

Definição: Seja $\mathrm{J}_{\mathrm{n}}$ um conjunto e seja $\sigma \in \mathrm{P}\left(\mathrm{J}_{\mathrm{n}}\right)$. Então $\sigma$ é chamado um ciclo de comprimento $\mathrm{k}$ se existem elementos $\mathrm{a}_{1}, \mathrm{a}_{2}, \ldots, \mathrm{a}_{\mathrm{k}} \in \mathrm{J}_{\mathrm{n}}$, tais que

$$
\sigma\left(a_{1}\right)=a_{2}, \sigma\left(a_{2}\right)=a_{3}, \ldots, \sigma\left(a_{k-1}\right)=a_{k}, \sigma\left(a_{k}\right)=a_{1} \text { e } \sigma(x)=x
$$

para todos os outros elementos $\mathrm{x} \in \mathrm{J}_{\mathrm{n}}, \operatorname{com} \mathrm{x} \neq a_{i}$, para $\mathrm{i}=1,2 \ldots, \mathrm{k}$. Nesse caso, escreve-se $\sigma=\left(a_{1}, a_{2}, \ldots, a_{k}\right)$.

Para facilitar a leitura, repetiremos a permutação da maneira matemática convencional:

$$
f_{1}=\left(\begin{array}{rrrrrrrccccc}
1 & 2 & 3 & 4 & 5 & 6 & 7 & 8 & 9 & 10 & 11 & 12 \\
1 & 4 & 7 & 10 & 2 & 5 & 8 & 11 & 3 & 6 & 9 & 12
\end{array}\right)
$$

Esta permutação é de ordem 5 e é constituída de 4 ciclos, dois com 5 elementos e dois com 1 .

Ciclo 1: $\sigma_{1}=(1)$

Ciclo 2: $\sigma_{2}=\left(\begin{array}{lllll}2 & 4 & 10 & 6 & 5\end{array}\right)$

Ciclo 3: $\sigma_{3}=\left(\begin{array}{lllll}3 & 7 & 8 & 11 & 9\end{array}\right)$

Ciclo 4: $\sigma_{4}=(12)$

Uma outra maneira de representar esses ciclos seria:

Ciclo 1: $\sigma_{1}=\left(\begin{array}{llllllllllll}1 & 2 & 3 & 4 & 5 & 6 & 7 & 8 & 9 & 10 & 11 & 12 \\ 1 & 2 & 3 & 4 & 5 & 6 & 7 & 8 & 9 & 10 & 11 & 12\end{array}\right)=I$

Ciclo 2: $\sigma_{2}=\left(\begin{array}{cccrcccccccc}1 & 2 & 3 & 4 & 5 & 6 & 7 & 8 & 9 & 10 & 11 & 12 \\ 1 & 4 & 3 & 10 & 2 & 5 & 7 & 8 & 9 & 6 & 11 & 12\end{array}\right)$

Ciclo 3: $\sigma_{3}=\left(\begin{array}{cccccccccccc}1 & 2 & 3 & 4 & 5 & 6 & 7 & 8 & 9 & 10 & 11 & 12 \\ 1 & 2 & 7 & 4 & 5 & 6 & 8 & 11 & 3 & 10 & 9 & 12\end{array}\right)$

Ciclo 4: $\sigma_{4}=\left(\begin{array}{cccccccccccc}1 & 2 & 3 & 4 & 5 & 6 & 7 & 8 & 9 & 10 & 11 & 12 \\ 1 & 2 & 3 & 4 & 5 & 6 & 7 & 8 & 9 & 10 & 11 & 12\end{array}\right)=I$

Lema: Toda permutação pode ser escrita como um produto de seus ciclos disjuntos. 
No exemplo anterior, percebe-se que :

$$
f_{1}=\sigma_{1}^{\circ} \sigma_{2}^{\circ} \sigma_{3}^{\circ} \sigma_{4}
$$

A escrita "resumida" dos ciclos facilita a visualização da permutação e o cálculo de sua ordem, como será detalhado a seguir.

\section{3. - Estudo de Messiaen com 32 elementos rítmicos}

A figura a seguir apresenta os elementos rítmicos ordenados de forma crescente. Os números acima, dentro de cada barra, correspondem ao total de fusas.

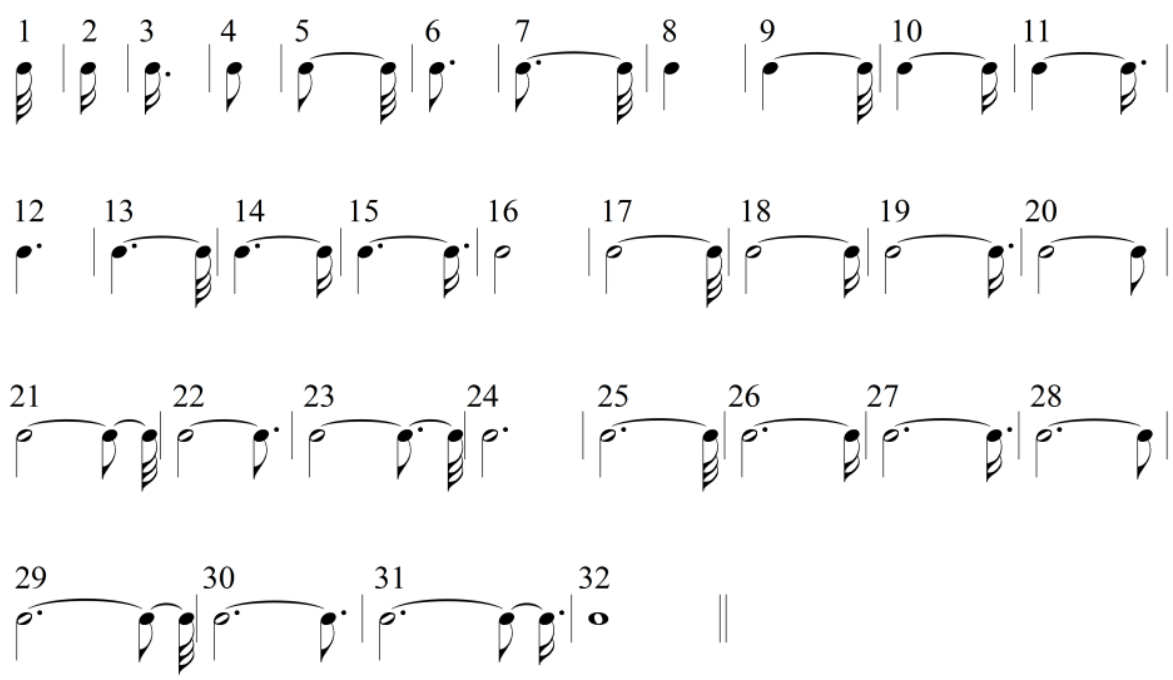

Figura 6: Representação numérica de um cromatismo rítmico com 32 elementos (Messiaen 1996, p.14)

A escolha da permutação feita por Messiaen foi a seguinte:

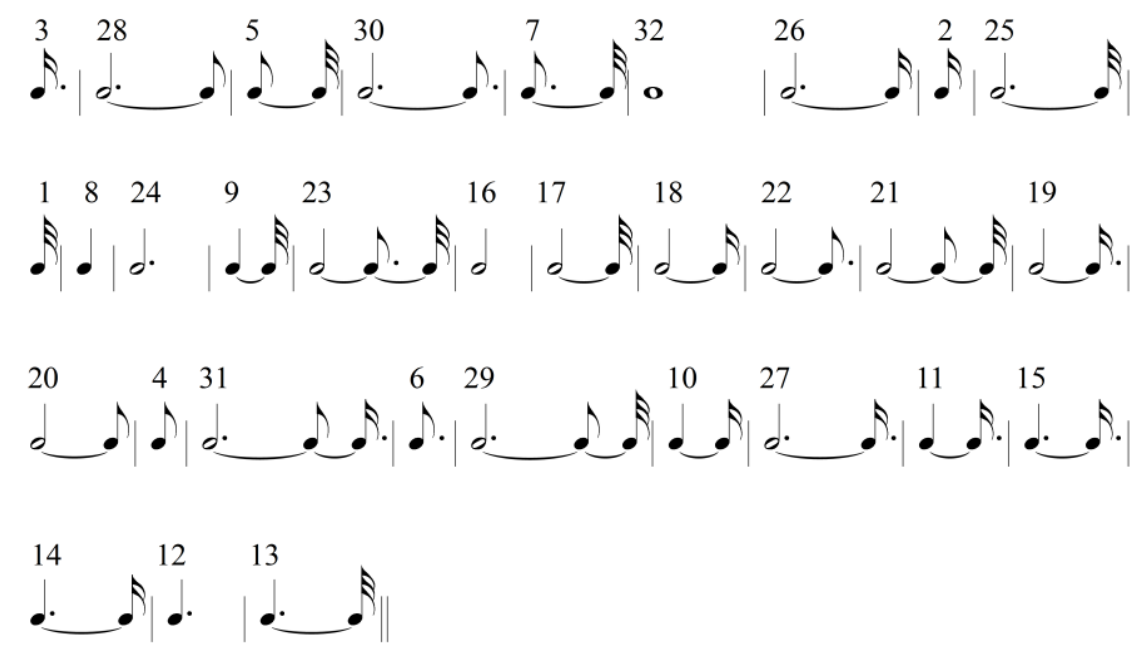

Figura 7: Permutação escolhida representada em figuras musicais

(Messiaen 1996, p.15) 
De acordo com a notação introduzida anteriormente, essa permutação ficaria da forma a seguir:

$\left(\begin{array}{llllllllllllllllllllllllllllllll}1 & 2 & 3 & 4 & 5 & 6 & 7 & 8 & 9 & 10 & 11 & 12 & 13 & 14 & 15 & 16 & 17 & 18 & 19 & 20 & 21 & 22 & 23 & 24 & 25 & 26 & 27 & 28 & 29 & 30 & 31 & 32\end{array}\right)$ $\left(\begin{array}{llllllllllllllllllllllllllllllllll}1 & 28 & 5 & 30 & 7 & 32 & 26 & 2 & 25 & 1 & 8 & 24 & 9 & 23 & 16 & 17 & 18 & 22 & 21 & 19 & 20 & 4 & 31 & 6 & 29 & 10 & 27 & 11 & 15 & 14 & 12 & 13\end{array}\right)$

Indiquemos essa permutação por $f$.

Compondo continuamente $f$, teremos que $f^{36}$ será a identidade: $f_{0}$, ou seja, a permutação escolhida é de ordem 36 .

Para calcular a ordem de uma permutação escolhida, observe o seguinte:

- começando pelo elemento 1, note que ele gera um ciclo, pois vai para o elemento 3 na primeira iteração, para o elemento 5 na segunda iteração, para o 7 na terceira, para o 26 na quarta, para o 10 na quinta e volta para o 1 na sexta, repetindo esse ciclo sucessivamente. Este primeiro ciclo, denotado por $\sigma_{1}$, é composto de 6 elementos: 1, 3, 5, 7, 26 e 10;

- o mesmo pode ser observado para os ciclos que se iniciam com os elementos 2 (ciclo 2: $\sigma_{2}$ ), 4 (ciclo 3: $\sigma_{3}$ ) e 19 (ciclo 4: $\sigma_{4}$ ), formando ciclos sucessivamente de 4 , 18 e 3 elementos;

- o elemento 27 do conjunto não sofre alterações porque faz "troca" somente com ele mesmo, ou seja, para qualquer $f^{n} \operatorname{com} n \in \mathbb{Z}$, o elemento 27 será o mesmo. Com isto podemos considerar que esse elemento possui um ciclo em torno de si mesmo, sem envolver os demais elementos. Ele forma o ciclo $5, \sigma_{5}$, de apenas 1 elemento.

Dessa forma podemos construir os seguintes ciclos de permutações:

Ciclo 1: $\sigma_{1}=\left(\begin{array}{llllll}1 & 3 & 5 & 7 & 26 & 10\end{array}\right)$

Ciclo 2: $\sigma_{2}=\left(\begin{array}{llll}2 & 28 & 11 & 8\end{array}\right)$

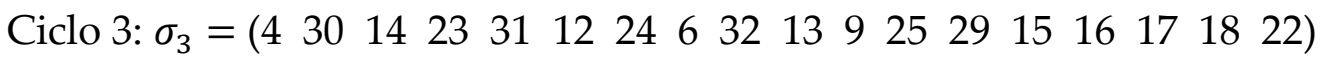

Ciclo 4: $\sigma_{4}=\left(\begin{array}{lll}19 & 21 & 20\end{array}\right)$

Ciclo 5: $\sigma_{5}=(27)$

Como a cada 6 iterações de $f$ o primeiro elemento do primeiro ciclo volta a ser 1 , o número de permutações para voltarmos a ter a identidade deve ser um múltiplo de 6.

$$
f^{m} / m \text { múltiplo de } 6 .
$$

Da mesma forma, temos que, para o segundo ciclo, o número de permutações aplicadas deve ser um múltiplo de 4, para o terceiro ciclo, um múltiplo de 18, para o quarto ciclo, um múltiplo de 3 e para o quinto ciclo um 
múltiplo de 1, pois o segundo ciclo é composto por 4 elementos, o terceiro por 18, o quarto por 3 e o quinto por 1 .

Então, para que voltemos à identidade, o número de iterações deve ser um múltiplo tal que chegue à identidade em todos os ciclos. Isto é, a ordem $n$ será dada por

$$
n=m \cdot m \cdot c(6,4,18,3,1)=36 \text {. }
$$

Portanto, $f^{36}=f_{0}=I$.

\section{4. - Aplicação musical da permutação}

Em sua obra Éclairs sur l'Au-delà..., peça para grande orquestra escrita entre 1987 e 1991, em onze movimentos, Messiaen sobrepõe de 3 em 3 as 36 permutações obtidas, gerando, dessa maneira, 12 sobreposições. A figura a seguir (Figura 8) ilustra a sobreposição gerada pelas permutações 16, 17 e 18.

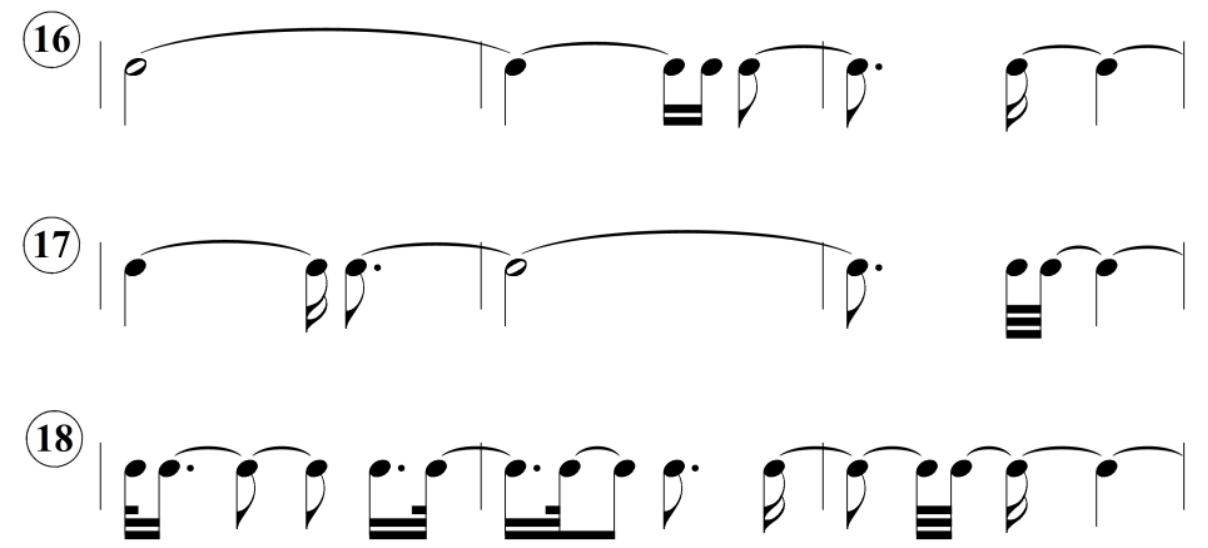

Figura 8: Sobreposição das permutações 16, 17 e 18, apenas início (Messiaen 1996, p.50)

A sobreposição demonstrada no exemplo anterior é utilizada no quarto movimento da peça, intitulado Les élus marqués du sceau. A permutação 16 é utilizada como ritmo de base para as linhas melódicas dos oito primeiros violinos junto com o primeiro jogo de sinos e três gongos agudos. A permutação 17 é tocada pelos oito segundos violinos, junto com o segundo jogo de sinos e três pratos. A permutação 18, por sua vez, é executada pelas violas, violoncelos, terceiro jogo de sinos e gongos graves (Figura 9). Junto a esta base harmônicorítmica criada com o auxílio das permutações, os instrumentos do naipe das madeiras tocam cantos de pássaros (não demonstrados na figura). 


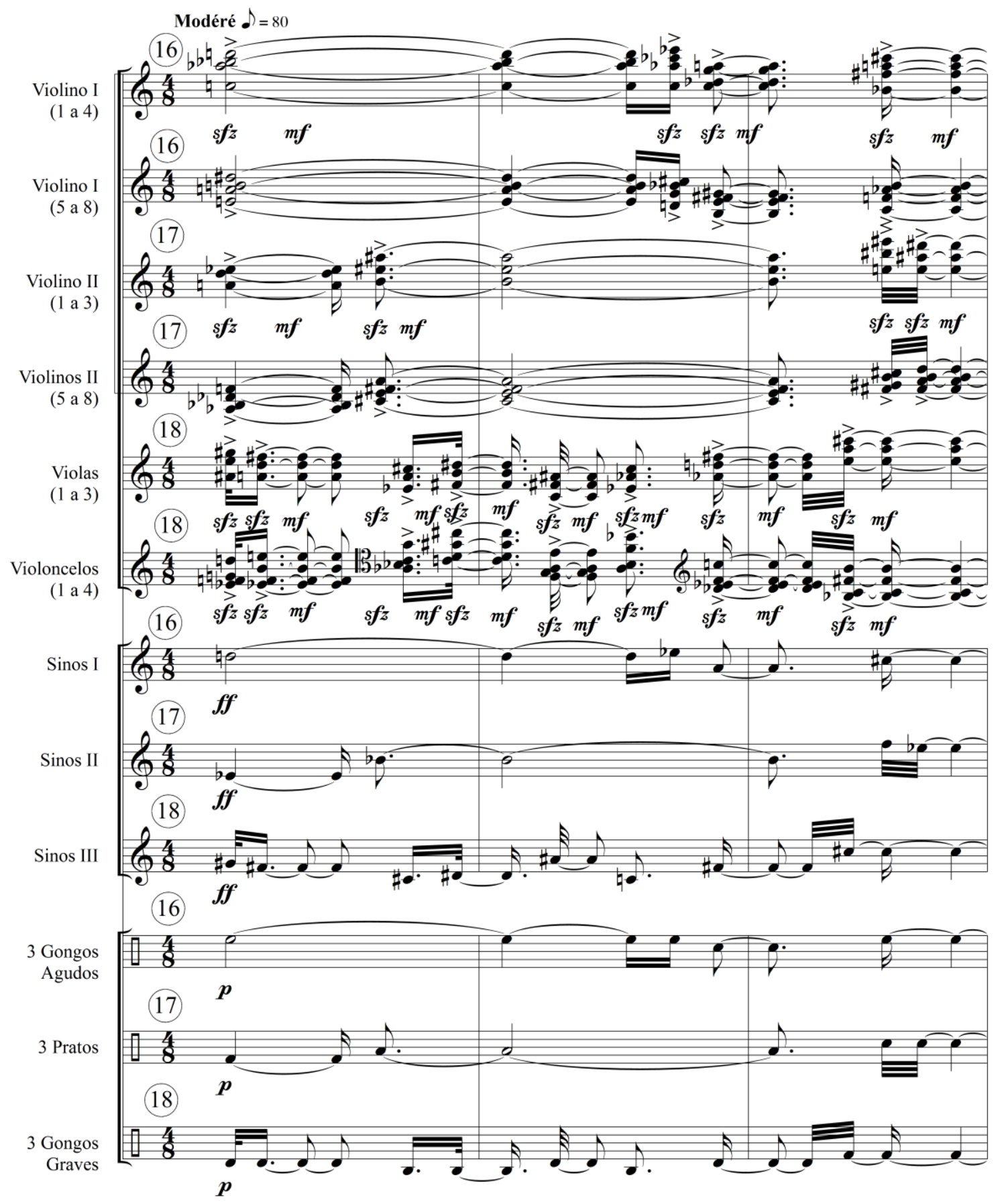

Figura 9: Sobreposição em instrumentos diversos das permutações 16, 17 e 18 nos três primeiros compassos do quarto movimento Les élus marqués du sceau, da peça Éclairs sur

l'Au-delà... (1987-1991) de Messiaen. Os instrumentos do naipe das madeiras estão omitidos na figura. (Messiaen 1996, p.74) 


\section{5. - Como obter uma permutação de ordem máxima}

Para se obter uma permutação de ordem máxima, deve-se conseguir o maior número de ciclos possível, com a quantidade de elementos primos entre si. No caso anterior, por exemplo, poderíamos conseguir 5 ciclos, com o seguinte número de elementos:

Ciclo 1: 3 elementos

Ciclo 2: 4 elementos

Ciclo 3: 5 elementos

Ciclo 4: 7 elementos

Ciclo 5: 13 elementos

Essa escolha levaria a uma permutação de ordem 5460, pois:

$$
\text { m.m.c }(3,4,5,7,13)=5460
$$

\section{6. - Considerações parciais}

Conforme detalhado anteriormente, Messiaen usa ritmicamente em suas composições uma única permutação que é iterada diversas vezes até retornar à identidade. Isto é o que ele chama de "Permutações Simétricas". Mas, qual a razão do termo "simétricas"? Antes de respondermos a esta indagação, investiguemos o que se entende por simetria sob a ótica da Geometria.

\section{3 - Simetria}

Antes de definir simetria, faz-se necessário primeiramente definir isometria (definição 1), porque a simetria é um tipo especial de isometria.

- Definição 1: $T: \mathbb{R}^{2} \rightarrow \mathbb{R}^{2}$ é uma isometria $\left(\right.$ de $\left.\mathbb{R}^{2}\right)$ se

$$
|T(z)-T(w)|=|z-w|
$$

quaisquer que sejam $z$ e $w$ de $\mathbb{R}^{2}$. A Figura 10Figura 10: Exemplo de uma isometria a seguir, ilustra esta função. 

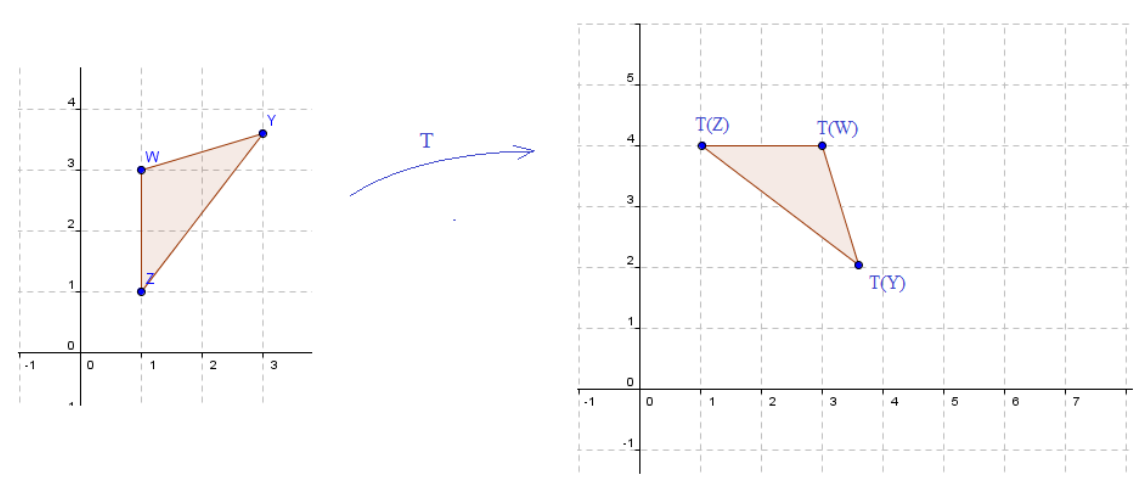

Figura 10: Exemplo de uma isometria

Observa-se que a isometria transforma a figura em outra de tal forma que a distância entre quaisquer de seus pontos é preservada. Na figura anterior, percebe-se, por exemplo, que o ponto $z$ é transformado em $T(z)$ e o ponto $w$ em $T(w)$, de tal maneira que a distância entre $z$ e $w$ é preservada. Matematicamente, dizemos que $|z-w|$ (a distância entre $z$ e $w$ ) é igual à $|T(z)-T(w)|$ (distância entre $T(z)$ e $T(w)$ ). Tratam-se de isometrias as translações, rotações e reflexões de figuras planas.

De posse dessa definição, pode-se agora definir simetria (definição 2).

- Definição 2: Uma simetria de uma figura F de um plano é definida como uma isometria $\mathrm{T}: \mathbb{R}^{2} \rightarrow \mathbb{R}^{2}$ que deixa a figura invariante ${ }^{1}$, isto é,

$$
T(F)=F .
$$

ou seja, uma simetria é uma isometria que transforma a figura nela mesma novamente. É importante frisar que nem toda isometria é uma simetria. Na Figura 10, por exemplo, a transformação ocorrida no triângulo da direita em relação ao da esquerda é uma isometria e não uma simetria, pois este foi transladado e rotacionado a uma posição do plano distinta da original.

A transformação $\mathrm{T}$ pode ser uma reflexão, uma translação ou uma rotação para ser uma simetria. Caso T seja uma reflexão, a simetria é dita simetria de reflexão; caso T seja uma translação, a simetria é dita simetria de translação e caso $\mathrm{T}$ seja uma rotação, a simetria é dita simetria de rotação. Essas três simetrias serão expostas nos itens a seguir.

\footnotetext{
${ }^{1}$ Adaptado de Bastos, 2006.
} 


\section{1. - Simetria de reflexão}

São aquelas cujas simetrias são obtidas a partir da reflexão em torno de um eixo.

\subsection{1. - Simetria de reflexão vertical}

É a mais utilizada no cotidiano. Também é chamada de simetria axial. Uma figura possui este tipo de simetria se pode ser refletida em relação a um eixo vertical (dito eixo de simetria), de modo a ser possível fazer-se corresponder ponto a ponto com a imagem original.

Por exemplo, a letra "A" possui uma simetria de reflexão vertical (Figura 11). Esta letra cobre exatamente o mesmo conjunto de pontos do plano se for refletida ao redor de um eixo vertical que passa pelo seu topo.

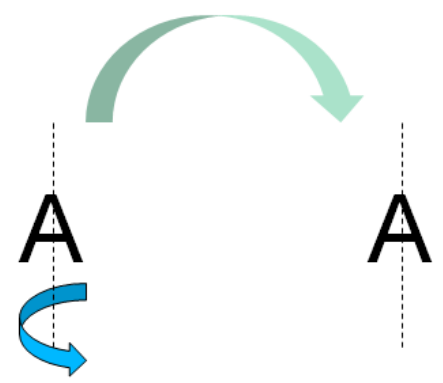

Figura 11: Simetria de reflexão da letra A

As letras $\mathrm{M}$ e $\mathrm{V}$ também possuem esse tipo de simetria.

\subsection{2. - Simetria de reflexão horizontal}

Uma figura possui este tipo de simetria se pode ser refletida em relação a um eixo horizontal de modo a ser possível fazer-se corresponder ponto a ponto com a imagem original. As letras C, E e K possuem esse tipo de simetria.

\section{2. - Simetria de translação}

Uma figura possui este tipo de simetria se existir uma translação que preserve a figura, ou seja, se pudermos movimentar a figura segundo uma dada distância e uma dada direção de tal modo que a figura transformada coincida com a original. Em caso afirmativo, a figura deve ser ilimitada. Supondo a Figura 12 , a seguir, infinita na direção do eixo $x$, percebe-se que esta possui uma simetria de translação, a qual é de comprimento $\overline{\mathrm{OA}}$ para a direita e uma simetria de 
translação de comprimento $\overline{\mathrm{OA}}$ para a esquerda. Além dessa, possui uma simetria de reflexão em torno do eixo Ox e também uma simetria de reflexão em torno do eixo Oy.

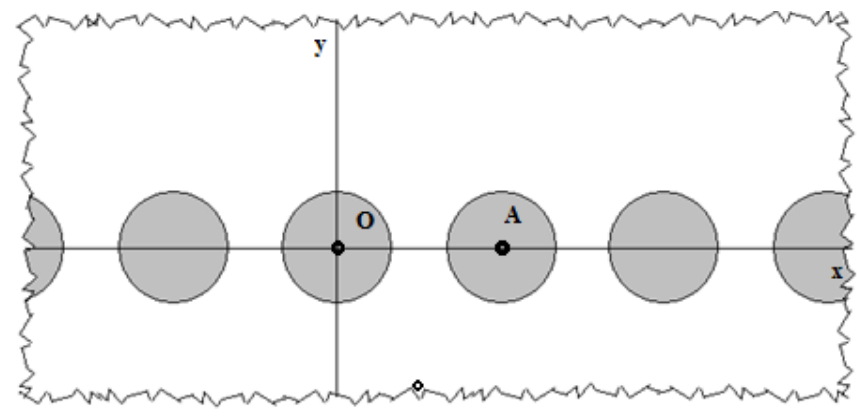

Figura 12: Simetria de translação

\section{3. - Simetria de rotação}

Outra simetria possível é a rotação ao redor de um ponto. Uma figura possui este tipo de simetria se existir uma rotação diferente da identidade que preserve a figura. A Figura 13, a seguir, é um exemplo deste tipo de simetria.

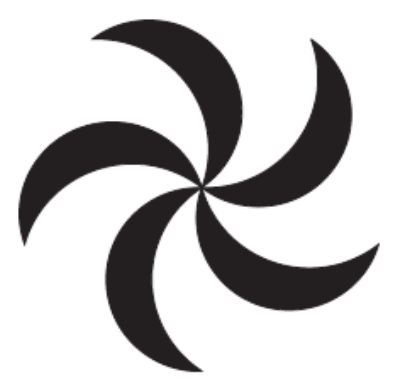

Figura 13: Simetria de rotação

Ao rodarmos esta figura de $72^{\circ}$ (ou $144^{\circ}$, ou $216^{\circ}$, ou $288^{\circ}$ ) em torno do seu ponto central, a figura resultante é a mesma que a original.

A letra Z é um outro exemplo (Figura 14). Ao rodar-se esta letra de 180 graus ao redor do seu ponto central, obtém-se novamente a mesma letra. Ao completar uma volta, ela volta a ser ela mesma duas vezes. Por isso, esta é considerada uma figura com rotação composta.

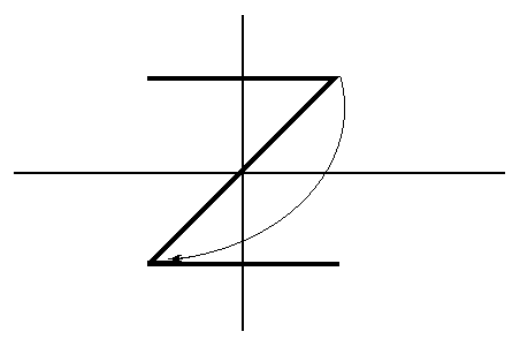

Figura 14: Simetria de rotação 
As letras $\mathbf{N}$ e $\mathbf{S}$ também possuem este tipo de simetria.

\section{4. - Considerações parciais}

Após havermos detalhado o que se entende por Simetria sob a ótica da Geometria e por Permutação, passemos agora a refletir sobre o termo usado por Messiaen, "Permutações Simétricas". Nomeadas desta maneira, é de se esperar que as permutações possuam propriedades que as designem simétricas, tais como a existência de um eixo de reflexão, ou de um parâmetro que se repete ao infinito (simetria de translação). Então, seriam realmente simétricas as permutações criadas por Messiaen?

\section{4 - Permutações simétricas e geometria}

Ligando a ideia de permutação a figuras geométricas, poderíamos ter, por exemplo, uma permutação simétrica em um pentágono regular, pois ele pode voltar a ser ele mesmo após uma simetria de rotação, no caso, de um ângulo igual a 72 graus, que é o seu ângulo interno. Observe a Figura 15 a seguir.
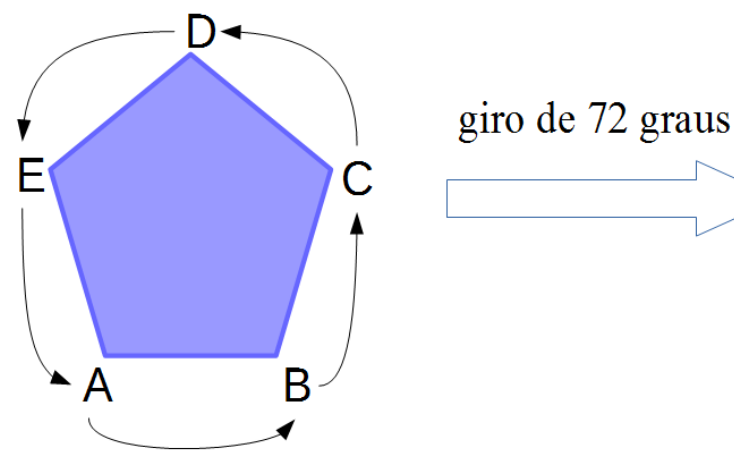

Figura 15: Pentágono original e pentágono rotacionado de 72 graus

Esta permutação (seja $f_{1}$ ) pode ser representada da seguinte maneira:

$$
f_{1}=\left(\begin{array}{lllll}
A & B & C & D & E \\
B & C & D & E & A
\end{array}\right)
$$

ou por meio de um único ciclo:

$$
\sigma_{1}=(A B C D E),
$$

o qual ainda pode ser representado da seguinte maneira (Figura 16):

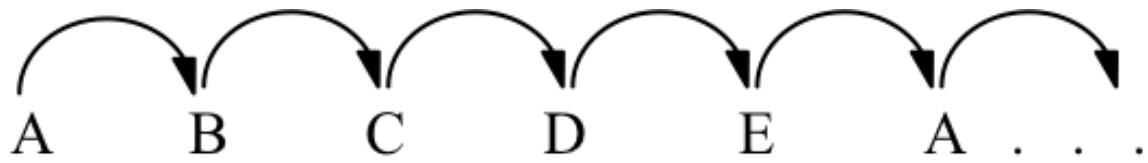

Figura 16: Simetria de translação 
Esta permutação é de ordem 5, pois é constituída de um único ciclo com 5 elementos. Todas as iterações seriam simétricas, pois resultariam no mesmo pentágono. Além disso, podemos comparar esta permutação a uma simetria de translação, pois cada vértice sempre é trocado pelo vértice seguinte, indefinidamente. Uma rotação de 72 graus no sentido horário, resultaria em uma outra permutação simétrica.

Poderíamos também obter permutações simétricas, por meio de simetrias de reflexão. Por exemplo (Figura 17):
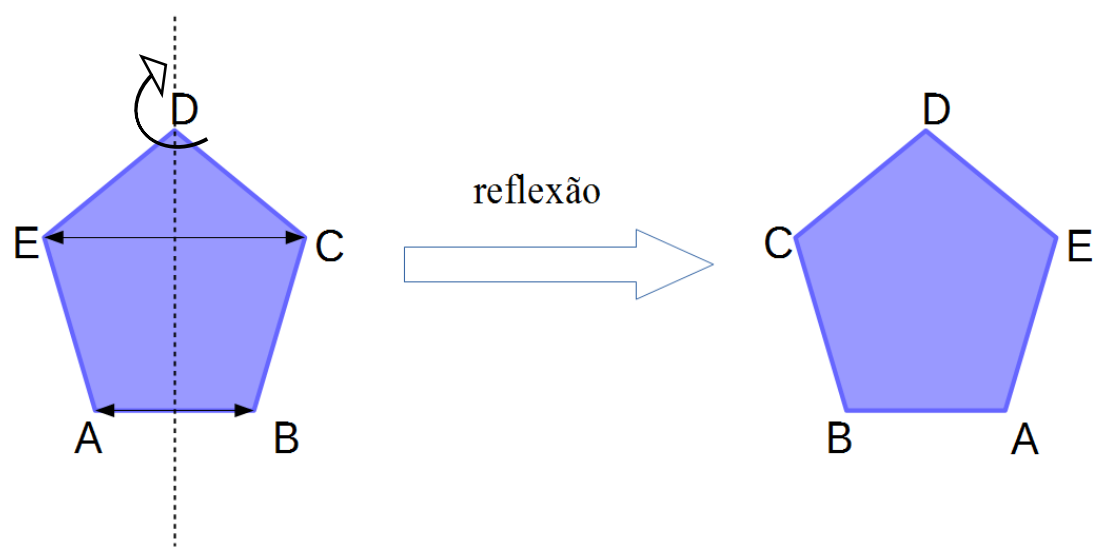

Figura 17: Simetria de reflexão

a qual foi obtida por meio de um eixo de reflexão passando pelo vértice $D$. Pode ser representada pela notação:

$$
f_{2}=\left(\begin{array}{lllll}
A & B & C & D & E \\
B & A & E & D & C
\end{array}\right)
$$

Esta permutação é de ordem 2, pois é constituída de 3 ciclos, dois com 2 elementos e um com 1.

Ciclo 1: $\sigma_{1}=(\mathrm{D})$

Ciclo 2: $\sigma_{2}=(\mathrm{C} \mathrm{E})$

Ciclo 3: $\sigma_{2}=(\mathrm{B}$ A)

Poderíamos representar esta permutação da seguinte maneira (Figura 18):

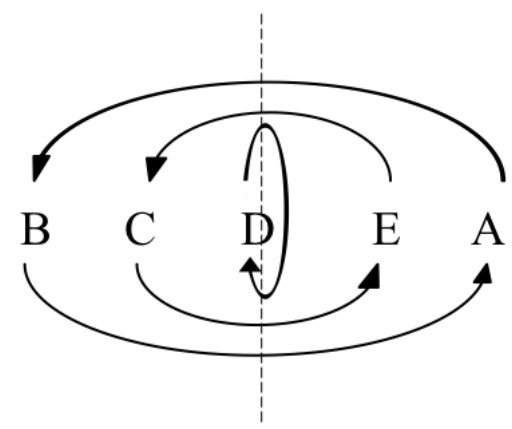

Figura 18: Simetria de reflexão, com eixo de simetria passando por D. 
Representada desta maneira, percebe-se uma simetria de reflexão nesta permutação, com o eixo de simetria passando por D.

Do mesmo modo, poderíamos ter mais quatro simetrias de reflexão, colocando eixos de reflexão passando pelos demais vértices.

Após estas outras maneiras de se representar uma permutação, retornaremos à permutação com 12 elementos de Messiaen apresentada na figura 2. É possível perceber que esta apresenta uma simetria de reflexão segundo um eixo que passa pelos elementos 6 e 7. É o que será apresentado a seguir.

Para facilitar a leitura, repetiremos a permutação da maneira matemática convencional:

$$
f_{1}=\left(\begin{array}{rrrrrrrccccc}
1 & 2 & 3 & 4 & 5 & 6 & 7 & 8 & 9 & 10 & 11 & 12 \\
1 & 4 & 7 & 10 & 2 & 5 & 8 & 11 & 3 & 6 & 9 & 12
\end{array}\right)
$$

Esta permutação é de ordem 5 , pois é constituída de 4 ciclos, dois com 5 elementos e dois com 1.

Ciclo 1: $\sigma_{1}=(1)$

Ciclo 2: $\sigma_{2}=\left(\begin{array}{lllll}2 & 4 & 10 & 6 & 5\end{array}\right)$

Ciclo 3: $\sigma_{3}=\left(\begin{array}{lllll}3 & 7 & 8 & 11 & 9\end{array}\right)$

Ciclo 4: $\sigma_{4}=(12)$

Poderíamos representar esta permutação da seguinte maneira:

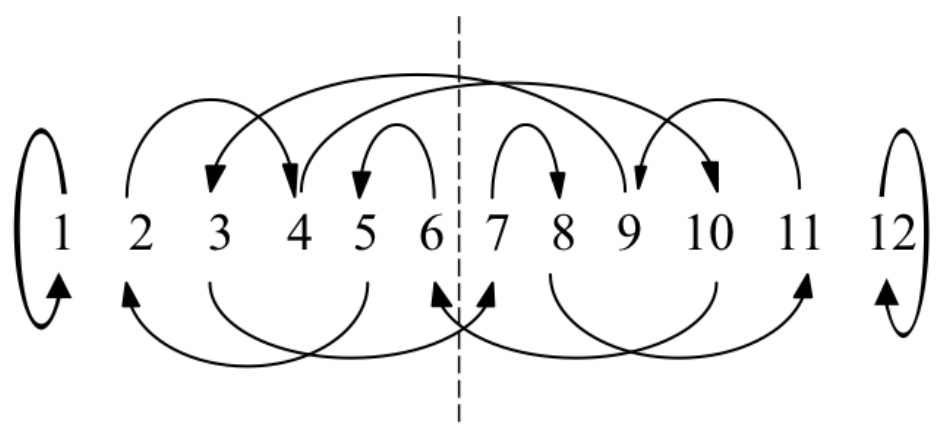

Figura 19: Simetria de reflexão, com eixo de simetria passando entre os elementos 6 e 7.

Percebe-se que esta tem uma simetria de reflexão em relação ao eixo que passa entre os elementos 6 e 7, conforme representado na Figura 19 acima. Poderíamos escrever matematicamente esta simetria por meio da equação:

$$
f_{1}(13-i)=13-f_{1}(i),
$$

onde i representa o i-ésimo elemento.

Uma outra simetria de reflexão pode ser encontrada em relação ao eixo que passa entre os elementos 12 e 1 (Figura 20). 


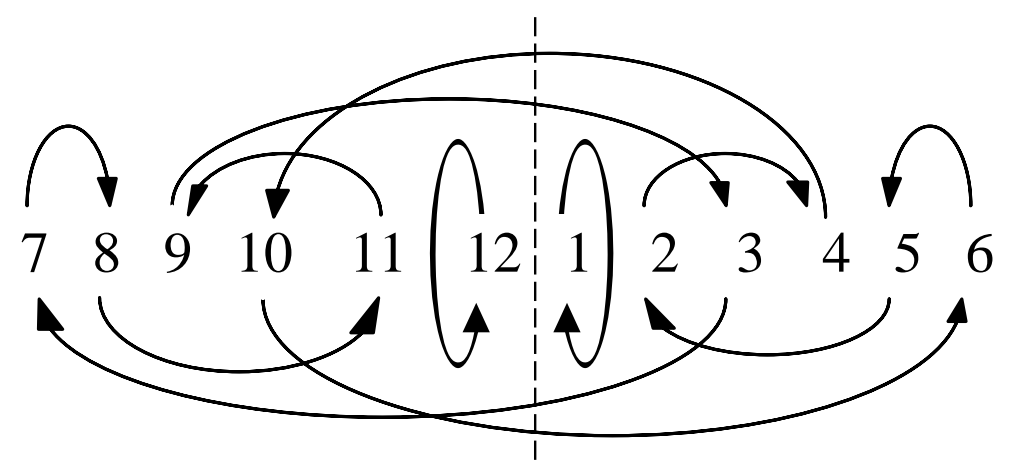

Figura 20: Simetria de reflexão, com eixo de simetria passando entre os elementos 12 e 1.

Retornando agora à permutação de Messiaen com 32 elementos rítmicos, a qual está reapresentada a seguir,

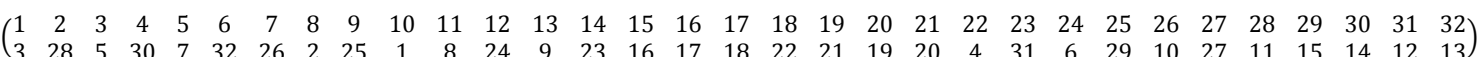

e a qual pode ser apresentada da seguinte forma (Figura 21):

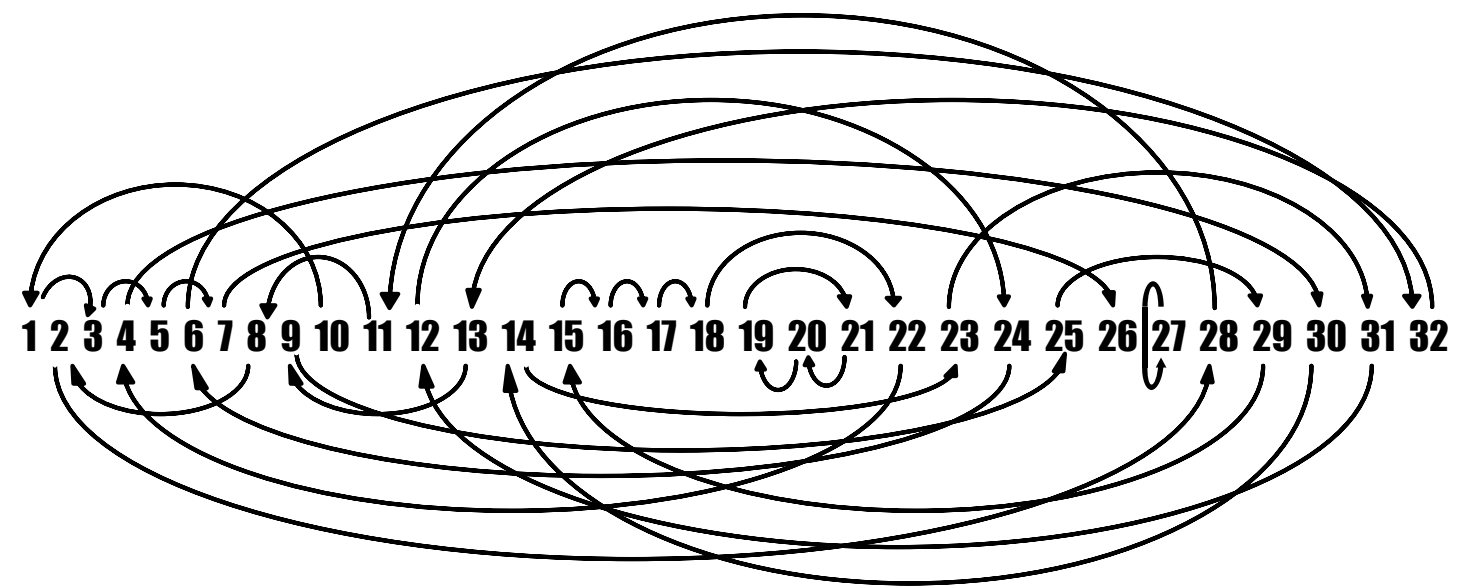

Figura 21: permutação de Messiaen com 32 elementos rítmicos

afirmamos que não é possível exibir qualquer eixo de simetria, pois o elemento 27 é o único elemento que o leva a ele mesmo. Assim, só poderíamos ter uma simetria de reflexão caso tivéssemos um eixo passando pelo próprio elemento 27. Entretanto, para que isso pudesse acontecer, deveríamos ter um número ímpar de elementos, o que, com 32 elementos, não acontece. Tampouco ocorrem simetrias de translação ou rotação nessa situação. 


\title{
5 - Considerações Finais
}

Percebe-se que, na prática composicional do compositor francês Olivier Messiaen havia a preocupação em encontrar uma maneira de compor que obedecesse certa lógica e que gerasse uma gama de possibilidades dentro de um conjunto limitado, objetivo esse que foi alcançado com o grupo de permutações. Por meio de tal grupo, obteve uma variedade de combinações rítmicas com a utilização de um número limitado de elementos. É o que ele considerava um "encantamento mágico" ${ }^{2}$, um "charme das impossibilidades"3: "certos circuitos fechados possuem um poder de fascínio, uma força mágica, um charme" 4 (Messiaen 1996, p. 7). Destacam-se nesse aspecto as Permutações Simétricas, abordadas no presente artigo, os Ritmos não Retrogradáveis e os Modos de Transposições Limitadas.

Segundo Sawatzky,

Messiaen, e vários comentaristas depois dele, consideram a técnica de Permutação Simétrica como uma maneira de selecionar um número relativamente pequeno e coerente de ordenações inter-relacionadas de $n$ elementos daquilo que, de outra forma, poderia ter sido uma enorme coleção de ordenações possíveis ${ }^{5}$ (Sawatzky 2013, p. 19).

A enorme coleção referida na citação anterior equivaleria a n! elementos.

Cheong comenta as limitações geradas pelo esquema de Permutação Simétrica da seguinte maneira:

\begin{abstract}
Assim como os modos de transposição limitada desfrutam menos que 12 transposições e os ritmos não retrogradáveis conservam o padrão original de duração quando tocados de trás para frente, um esquema de Permutação Simétrica também pode cortar drasticamente o número de permutações deriváveis de qualquer série dada ${ }^{6}$ (Cheong, apud Sawatzky 2013, p. 19)
\end{abstract}

\footnotetext{
2 "Enchantement magique".

3 "Charme des impossibilités".

4 "certains circuits fermés, possèdent une puissance d'envôutement, une force magique, un charme".

5 "Messiaen, and many commentators after him, position the SP technique as a way of selecting a coherent and relatively small number of interrelated orderings of $n$ elements from what would have otherwise been an enormous collection of all possible orderings".

6 "Just as the modes of limited transposition enjoy fewer than twelve transpositions and the nonretrogradable rhythm retains the original duration pattern when played backward, a symmetrical permutation scheme can also drastically cut down the number of permutations derivable from any given series".
} 
Apesar de Messiaen chamar as Permutações de simétricas, o que se percebe é que estas não têm, necessariamente, as propriedades que as designariam simétricas, em termos geométricos, pois não possuem obrigatoriamente simetrias de reflexão, de translação e/ou de rotação. Notamos, entretanto, que há simetria em algumas das Permutações escolhidas, tal como a simetria de reflexão encontrada no estudo com 12 elementos demonstrado nas Figura 18 e Figura 19. A tese defendida no presente artigo de que Messiaen não pensava obrigatoriamente em permutações com simetrias é reforçada pelo fato de não termos encontrado simetria de tipo algum na permutação de 32 elementos.

Nesse trabalho, a partir de uma proposição advinda de um músico, foi possível buscar respostas e soluções por meio da Matemática. Talvez sem se dar conta da utilização da ferramenta matemática Grupo de Permutações, Messiaen conseguiu limitar em seus trabalhos as possibilidades rítmicas de sua obra, o que, para ele, é enriquecedor para a sua prática composicional. Revela-se, dessa maneira, a importância de se ter uma visão interdisciplinar, sem fronteiras sólidas entre as disciplinas, rompendo as suas próprias lógicas, enriquecendo e indo além de conhecimentos específicos.

\section{Referências}

1. Beachy, John A.; Blair, William D. 1996. Abstract Algebra. Long Grove, Illinois: Waveland Press.

2. Dean, Richard A. 1974. Elementos de Álgebra Abstrata. Rio de Janeiro: Livros Técnicos e Científicos.

3. Herstein, Israel N. 1996. Abstract Algebra. 3a ed. Upper Saddle River: Prentice Hall.

4 . 1975. Topics in Algebra. 2ª ed. New York: John Willey \& Sons.

5. Iezzi, Gelson; Dolce, Osvaldo 1973. Álgebra III. São Paulo: Moderna.

6. Messiaen, Olivier 1996. Traité de rythme, de couleur, et d'ornithologie. Tome III. Paris: Alphonse Leduc.

7. Sawatzky, Grant. M. 2013. Olivier Messiaen's Permutations Symétriques in Theory and Practice. Master of Arts. Vancouver: University of British Columbia.

8. Souza, Luciana Gastaldi S. 2012. Uma abordagem didático-pedagógica da racionalidade matemática na criação musical. Tese de doutorado. São Paulo: USP. 